\title{
Relação entre parâmetros do sistema oxidativo e distintos escores de claudicação em vacas leiteiras
}

Adelina Rodrigues Aires ${ }^{[a]}$, José Francisco Xavier da Rocha ${ }^{[a]}$, Felipe Lamberti Pivoto ${ }^{[b]}$, Sergio Henrique Mioso Cunha ${ }^{[c]}$, Rafael Costa Ebling ${ }^{[b]}$, Alexandre Krause ${ }^{[c]}$, Marta Lizandra do Rêgo Lea ${ }^{[b]^{*}}$

\footnotetext{
[a] Departamento de Medicina Veterinária, Universidade do Oeste de Santa Catarina (UNOESC), São Miguel do Oeste, SC, Brasil ${ }^{[b]}$ Departamento de Clínica de Grandes Animais, Laboratório de Endocrinologia e Metabologia Animal, Universidade Federal de Santa Maria (UFSM), Santa Maria, RS, Brasil

${ }^{[c]}$ Medicina Veterinária, Faculdades de Itapiranga (FAI), Itapiranga, SC, Brasil

[d] Departamento de Clínica de Pequenos Animais, Laboratório de Patologia Clínica Veterinária, Universidade Federal de Santa Maria (UFSM), Santa Maria, RS, Brasil
}

*Autor correspondente

e-mail: martalizandra@gmail.com

\section{Resumo}

A claudicação é uma das maiores causas de descartes, perdas econômicas e comprometimento no bemestar animal de bovinos leiteiros. No entanto, a patogenia envolvida nas doenças podais ainda é pouco explorada. Dessa forma, o objetivo deste estudo foi avaliar o papel das espécies reativas ao oxigênio e de enzimas do sistema antioxidante em vacas leiteiras com diferentes graus claudicação. Foram avaliadas 56 vacas Holandesas, sendo 28 oriundas de fazenda localizada em Cachoeira do Sul/RS, e 28 provenientes de propriedade leiteira situada em Santo Augusto/RS. Os animais foram selecionados e agrupados de acordo com o escore de claudicação (EC1, EC2, EC3 e EC4). Amostras de sangue foram coletadas, em um único momento experimental, para determinação dos teores de espécies reativas ao ácido tiobarbitúrico (TBARS), da atividade das enzimas superóxido dismutase (SOD) e glutationa peroxidase (GPx), e dos teores séricos de fibrinogênio. Maiores valores de TBARS foram detectados nos animais com EC3 e EC4 em relação àqueles com EC1. Observou-se maior atividade da GPx nos animais do grupo EC3 em comparação aos grupos EC1 e EC2. Valores médios mais elevados da SOD foram observados nos animais com EC4 em relação àqueles com escore de claudicação um (EC1). Não houve diferença nos teores séricos de fibrinogênio entre os grupos. Embora maiores valores de TBARS tenham sido detectados nos bovinos com EC3 e EC4, observouse uma resposta compensatória das enzimas antioxidantes (GPX e SOD) nestes animais. Os resultados indicam que não houve estresse oxidativo nos bovinos com distintos escores de claudicação, possivelmente 
em decorrência de um incremento na resposta antioxidante enzimática, a fim de evitar possíveis danos celulares causados pelo excesso na produção de espécies reativas de oxigênio (ERO) e de nitrogênio (ERN). 